\title{
Nanoporous GaN/n-type GaN: a cathode structure for ITO-free perovskite solar cells
}

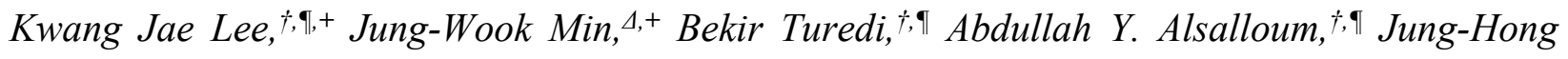
Min, ${ }^{4}$ Yeong Jae Kim, ${ }^{\circ}$ Young Jin Yoo, ${ }^{\circ}$ Semi Oh, $\square$ Namchul Cho, ${ }^{+}$Ram Chandra Subedi, ${ }^{\triangle}$ Somak Mitra, $\nabla$ Sang Eun Yoon, " Jong Hyun Kim, " Kwangwook Park, § Tae-Hoon Chung," Sung Hoon Jung, "Jong H. Baek, ${ }^{\#}$ Young Min Song, ${ }^{\circ}$ Iman S. Roqan, ${ }^{\nabla}$ Tien-Khee Ng, ${ }^{4}$ Boon S. Ooi, ${ }^{*}, 4$ and Osman M. Bakr ${ }^{*}+, \uparrow$

$\dagger$ Division of Physical Sciences and Engineering, "KAUST Catalyst Center (KCC), ${ }^{\Delta}$ Photonics Laboratory, Computer, Electrical and Mathematical Sciences and Engineering Division and $\nabla$ Semiconductor and Material Spectroscopy Laboratory, Material Science \& Engineering Division, King Abdullah University of Science and Technology (KAUST), Thuwal 23955-6900, Kingdom of Saudi Arabia

Department of Energy Systems Engineering, Soonchunhyang University, Asan 31538, Republic of Korea

${ }^{\circ}$ School of Electrical Engineering and Computer Science and $\square$ School of Materials Science and Engineering, Gwangju Institute of Science and Technology, Gwangju 61005, Republic of Korea \#Light Source Research Division, Korea Photonics Technology Institute (KOPTI), Gwangju 61007, Republic of Korea

" Department of Molecular Science and Technology, Ajou University, Suwon 16499, Republic of Korea

§Division of Advanced Materials Engineering, Jeonbuk National University, Jeonju 54896, Republic of Korea

AUTHOR INFORMATION

Corresponding Authors

*E-mail: osman.bakr@kaust.edu.sa (Osman M. Bakr), boon.ooi@kaust.edu.sa (Boon S. Ooi) 
Notes

+These authors contributed equally to this work.

\begin{abstract}
Introducing suitable electron/hole transport layers and transparent conductive layers (TCLs) into perovskite solar cells (PSCs) is key to enhancing the selective extraction of charge carriers and reducing surface recombination losses. Here, we introduce nanoporous gallium nitride (NP GaN)/n-type GaN (n-GaN) as a dual-function cathode structure for PSCs, acting as both the TCL and the electron transport layer (ETL). We demonstrate that the hierarchical NP GaN structure provides an expanded interfacial contact area with the perovskite absorber, while the $n-G a N$ under the NP GaN displays high transmittance in the visible spectrum as well as higher lateral electric conductivity than that of a conventional ITO film. Prototype $\mathrm{MAPbI}_{3}$ PSCs based on this NP GaN/n-GaN cathode structure (without an extra ETL) show a power conversion efficiency of up to $18.79 \%$. The NP GaN/n-GaN platform demonstrated herein paves the way for PSCs to take advantage of the widely available heterostructures of mature III-nitride-based technologies.
\end{abstract}

\title{
TOC GRAPHICS
}

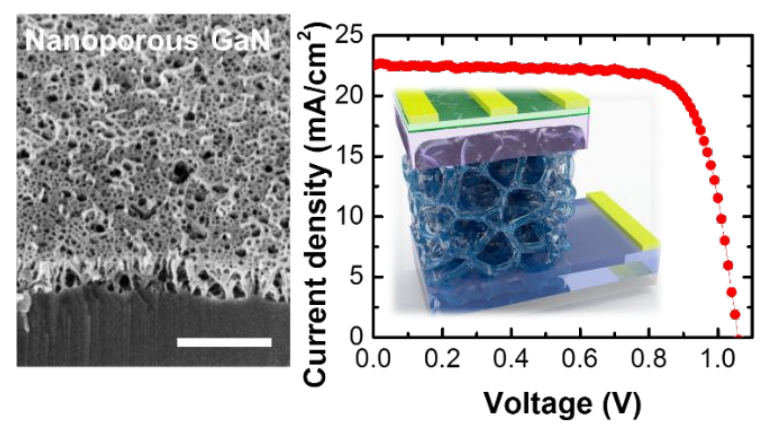


Halide perovskites with the general formula $\mathrm{AMX}_{3}$ (where $\mathrm{A}=\mathrm{CH}_{3} \mathrm{NH}_{3}{ }^{+}, \mathrm{CH}\left(\mathrm{NH}_{2}\right)_{2}{ }^{+}, \mathrm{Cs}^{+} ; \mathrm{M}=$ $\left.\mathrm{Pb}^{2+}, \mathrm{Sn}^{2+} ; \mathrm{X}=\mathrm{I}^{-}, \mathrm{Br}^{-}, \mathrm{Cl}^{-}\right)$have surged to the forefront of optoelectronic materials primarily due to the outstanding performance of perovskite solar cells (PSCs). ${ }^{1-8}$ Bandgap tunability (1.5 - 3.1 $\mathrm{eV})$, a high absorption coefficient $\left(10^{4}-10^{5} \mathrm{~cm}^{-1}\right)$, low exciton binding energy $(<50 \mathrm{meV})$, and simplicity of fabrication, are some of the few properties that played a key role in cementing the place of halide perovskites among solar cell materials. ${ }^{9-14}$ However, because dopant-host interactions in perovskites are distinct from those of traditional semiconductor materials (for example, Si:B, GaAs:Mg, and AlN:Si), ${ }^{15-17}$ perovskites have not yet been formed into $p-i-n$ junctions through doping. Thus, heterogeneous materials need to be integrated to create junctions that promote the charge extraction and collection in PSCs. In this respect, one of the critical issues concerning the fabrication of PSCs is designing the most suitable cathode structure by combining key material components for the extraction of photogenerated electrons, such as an electron transport layer (ETL) and a transparent conductive layer (TCL, cathode electrode).

In PSC cathodes, the ETL is specially designed not only to extract photocurrent but also to exhibit chemical and physical properties commensurate with those of the rest of the PSC structure, such as chemical durability, interfacial properties, thermal/electrical conductivities, bandgap alignment, and optical properties (e.g., refractive index and transmittance). ${ }^{18-20} \mathrm{TiO}_{2},{ }^{19} \mathrm{ZnO},{ }^{20}$ $\mathrm{SnO}_{2},{ }^{21}$ and $\mathrm{SrSnO}_{3}{ }^{22}$ are the best-known starting materials for forming ETLs; however, these wide-bandgap oxide materials have several disadvantages, which may include: poor electron mobility, ${ }^{19-22}$ chemical instability in acid/base solutions, ${ }^{23}$ reactivity with the perovskite layer, ${ }^{23}$ and high temperature processing, ${ }^{24,25}$ as well as difficulty in tuning band structure, particle size, and film morphology. ${ }^{26}$ 
Besides the ETL, a TCL such as indium tin oxide (ITO), is an essential part of a PSC for extracting photogenerated charge carriers from the perovskite solar absorber. ${ }^{2,27,28}$ The two requirements for a suitable TCL are high transmittance, to deliver more light to the perovskite absorber, and high electrical conductivity, to extract photogenerated electrons more efficiently through the horizontal current path. ${ }^{28}$ Unfortunately, during the last decade, the price of ITO has continuously risen due to the scarcity of indium, which may be depleted within the next decade. ${ }^{29-}$ 31

Gallium nitride $(\mathrm{GaN})$ possesses excellent optical and electrical properties due to its direct bandgap $\left(E_{\mathrm{g}} \sim 3.4 \mathrm{eV}\right)$, transmittance exceeding $82 \%$ over the entire visible wavelengths, and high electron mobility $\left(\sim 1,000 \mathrm{~cm}^{2} / \mathrm{Vs}\right) .{ }^{32}$ Facile control over the doping type at the desired carrier concentrations and the ease of $E_{\mathrm{g}}$ tuning with alloys $\left(\mathrm{Ga}_{x} \mathrm{Al}_{y} \operatorname{In}_{1-x-y} \mathrm{~N}\right)$ allow for versatile tailoring of GaN's electrical and optical characteristics. ${ }^{33}$ Furthermore, the thermal, mechanical, and chemical stability of GaN enable practical applications of light-emitting diodes (LEDs), ${ }^{34,35}$ laser diodes (LDs), ${ }^{36}$ and photodetectors (PDs). ${ }^{37}$ These desired characteristics have attracted researchers to study the combination of $\mathrm{GaN}$ with perovskites for new concepts of device applications such as piezo-phototronics, photovoltaics, and nanostructured LDs. ${ }^{38-42}$

$\mathrm{GaN}$ is generally grown by metal-organic chemical vapor deposition (MOCVD) ${ }^{43}$, hydride vapor phase epitaxy (HVPE) ${ }^{44}$ molecular beam epitaxy $(\mathrm{MBE}),{ }^{45}$ or one of other analogous pathways. ${ }^{46,47}$ The latest growth technologies have demonstrated their capacity for mass producing GaN wafers (> 12 inches) with high throughput, low fabrication costs, and the ability to grow films not only on monocrystalline substrates but also on non-crystalline substrates such as glass and flexible polymer substrates. ${ }^{48-50}$ Compared to existing commercialized cathode structures, the price of GaN/substrate is competitive and is likely to decrease with further adoption (Table S2). 
While some studies have demonstrated potential advantages of GaN for perovskite technology such as improved stability ${ }^{38}$ or the use of $\mathrm{GaN}$ as an ETL material ${ }^{39-41}$ in current device architectures - however, it is by eliminating undesirable heterogeneous interfaces, simplifying the device fabrication process, and expanding the design versatility of perovskite devices that GaN, arguably, could have the largest impact (by taking advantage of its versatile doping in combination with its monolithic growth processes).

Motivated by this goal, we introduce nanoporous (NP) GaN epitaxially grown on $n$-type GaN $(n-\mathrm{GaN})$ - referred to here as (NP GaN/n-GaN) - as a dual-function cathode structure containing both the TCL and the ETL for spin-coated methylammonium lead iodide $\left(\mathrm{MAPbI}_{3}\right)$ PSCs. Our finite-difference time-domain (FDTD) and rigorous coupled-wave analysis (RCWA) simulations show that this structure enhances photon harvesting in the cell due to the gradual change in the effective refractive index through the $\mathrm{NP} \mathrm{GaN} / \mathrm{MAPbI}_{3}$ layer. While one would expect that the increased interfacial contact area between the $\mathrm{NP} G a N$ and the $\mathrm{MAPbI}_{3}$ may also increase the probability that interface defects form, which could militate the device performance, a spontaneously forming $\mathrm{Ga}_{2} \mathrm{O}_{3}$ layer on the surface of the NP GaN (during the electrochemical etching-fabrication of the NP GaN layer) acts as an interlayer between the perovskite and NP GaN and, thereby, passivating defect-related deep levels. To illustrate these advantages, we demonstrate prototype $\mathrm{MAPbI}_{3}$ PSCs based on this NP GaN/n-GaN cathode structure (without any other ETL) with promising power conversion efficiencies (PCEs) of up to $18.79 \%$. Our work shows that IIInitride-based nanostructure can be versatile components in perovskite-based optoelectronics, enabling new device architectures that do away with rate limiting material layers.

To fabricate NP GaN, the electrochemical etching of $n$-GaN was conducted as shown in Figure 1a. $10 \mu \mathrm{m}$-thick $n$-GaN thin films were grown on the double-polished sapphire substrate 
to ensure high transmittance to visible light. Figure $\mathbf{1 b}$ displays the 2-inch wafers of as-grown $n$ $\mathrm{GaN}$ and NP GaN formed by $1 \mathrm{~min}, 5 \mathrm{~min}$, and $10 \mathrm{~min}$ of electrochemical etching at an applied bias of $12 \mathrm{~V}$ (Figure S1). By increasing the etching time, the transmittance of GaN (Figure 1c) was systematically decreased, and the integrated photoluminescence (PL) intensity (Figure 1d) was increased accordingly, due to the scattering effect from the formed nanopores. Figure 1e shows optical and confocal microscopy images of nanopores under a luminous source (450 $\mathrm{nm})$. Photons penetrating the NP GaN interact with the nanopores $\left(D_{\text {avg }}=37.5 \mathrm{~nm}\right)$, leading to Rayleigh scattering. This optical property of NP GaN causes the trajectory of photons to fluctuate, contributing to the enhancement in the light harvesting of the NP GaN medium. In terms of electrical properties, the current flow through NP GaN (denoted as A-A') was influenced by etching; the resistance was increased by increasing the etching time for the NP GaN structure (Figure 1f) due to the pore volume fraction, including pore interconnection and tortuosity. ${ }^{51}$ On the other hand, lateral electrical transport through the $n$-GaN/n-GaN structure (denoted as B-B') showed nearly the same resistivity regardless of the etching time (Figure 1g). Despite the presence of a porous layer along the direction of current flow, the NP GaN structure exhibited a lateral electric conductivity higher than that of a conventional ITO film (Figure 1h). Thus, the NP GaN/nGaN structure serves as a dual-function layer exhibiting properties of both a TCL and an ETL.

To explore the possibility of depositing a perovskite film on NP GaN by spin-coating of a perovskite solution, we conducted contact angle measurements. We calculated the surface area fraction $\left(\rho_{\mathrm{s}}\right)$ of NP GaN using plan-view scanning electron microscopy (SEM) images (Figure 2a). Figure $2 \mathbf{b}$ shows the contact angles measured for deionized water as a function of $\rho_{\mathrm{s}}$. The hydrophobicity of the dense $n$-GaN film was indicated by a large contact angle of $57.8^{\circ}$. Increasing the value of $\rho_{\mathrm{s}}$ of NP GaN caused the contact angle to decrease (Figure S2), with angles of $34.2^{\circ}$, 
$12.6^{\circ}$, and $7.3^{\circ}$ measured at $\rho_{\mathrm{s}}$ values of $7.2 \%, 47.5 \%$, and $71.7 \%$, respectively; this trend clearly reflects the hydrophilicity induced by introducing nanopores into the GaN surface. This result is attributed to the surface roughness and oxidation of $\mathrm{GaN}$ surface to $\mathrm{Ga}_{2} \mathrm{O}_{3}\left(\right.$ or $\left.\mathrm{GaO}_{3}{ }^{3-}\right)$ obtained by electrochemical etching, which induces higher surface tension energy than that observed for an intrinsic dense $n$-GaN surface. ${ }^{52}$ After ozone plasma treatment for 10 min, we spin-coated $\mathrm{MAPbI}_{3}$ perovskite onto a 2-inch NP GaN wafer (Figure 2c). Figure 2d and 2e shows how the morphology of the $\mathrm{MAPbI}_{3}$ perovskite films varied with the $\rho_{\mathrm{s}}$ of NP GaN as a result of changes in the spincoating conditions. At high porosities $\left(\rho_{\mathrm{s}}>71.7 \%\right)$, the perovskite film was embedded in the NP GaN; however, at low porosities $\left(\rho_{\mathrm{s}}<47.5 \%\right)$, the perovskite film was separated from the NP GaN. These results show that the perovskite could be confined in the nanopores when the $\rho_{\mathrm{s}}$ of the NP GaN layer was sufficiently high $\left(\rho_{\mathrm{s}}>71.7 \%\right)$. To summarize, NP GaN exhibits the prerequisite optical, electrical, and structural characteristics to be a promising substitute for the current layers employed as ETLs/TCLs in PSC devices.

To confirm that the NP GaN structure can serve as an alternative to conventional ETLs and TCLs, we fabricated proof-of-concept $\mathrm{MAPbI}_{3}$ PSCs based on this structure. Figure 3a shows a schematic illustration of a PSC featuring a NP GaN/MAPbI ${ }_{3}$ heterostructure. Using planar $n-\mathrm{GaN}$, NP GaN was formed by electrochemical etching for different durations. To identify the various layers of the solar cell structure, we cross-sectioned the structure by focused-ion-beam (FIB) processing and then probed the cross-section using transmission electron microscopy (TEM). Figure 3b shows corresponding cross-sectional high-angle annular dark-field scanning transmission microscopy (HAADF-STEM) images. The various layers of the solar cell are visually distinct, and both the planar GaN layer and perovskite-coated NP GaN are clearly shown. In STEM mode, electron energy loss spectroscopy (EELS) with element mapping of $\mathrm{N}, \mathrm{Ga}$, and $\mathrm{Au}$ 
confirmed that NP GaN structure remained well intact after solar cell fabrication. Elemental mapping of I and $\mathrm{Pb}$ revealed that the perovskite material was well-incorporated deep into the NP GaN structure (Figure S3). Additional EELS mapping for other elements was also performed at the interface of the pristine NP GaN layer without perovskite (Figure 3c). Note that in the area around the interface between the NP GaN and planar GaN layers, the constituent elements $(\mathrm{O}, \mathrm{Ga}$ and $\mathrm{N}$ ) are distributed uniformly throughout the topmost GaN surface of the NP GaN structure. Interestingly, a highly intense peak corresponding to $\mathrm{O}$ was found at the edge of the porous structures, suggesting that the $\mathrm{Ga}_{2} \mathrm{O}_{3}$ interlayer was formed as an outer shell around the NP GaN. This $\mathrm{Ga}_{2} \mathrm{O}_{3}$ likely resulted from the oxalic acid used during the electrochemical etching of NP GaN.

To better understand the role of $\mathrm{Ga}_{2} \mathrm{O}_{3}$ in NP GaN, we performed a series of optical characterizations on NP GaN samples (with and without $\mathrm{Ga}_{2} \mathrm{O}_{3}$ ). To fabricate NP GaN without $\mathrm{Ga}_{2} \mathrm{O}_{3}$, the sample was cleaned with acetone and isopropanol (IPA), then rinsed with deionized water in advance. Additional oxides were removed by dipping the sample in buffered oxide etch (BOE) for 5 min. Using a planar GaN layer as a reference, NP GaN samples were examined by low-temperature (10 K) PL measurements to confirm the crystallinity of GaN and analyze several defect states of the NP structures. Figure 3d shows neutral donor bound exciton $\left(\mathrm{D}^{\circ} \mathrm{X}\right)$ peaks around $3.478 \mathrm{eV}$ detected in three samples, which represent the dominant emission spectrum of GaN. These peaks agree well with the $\mathrm{D}^{\circ} \mathrm{X}$ peaks reported previously for MOCVD planar GaN layer templates grown on a sapphire substrate. ${ }^{52}$ It should be noted that $\mathrm{D}^{\circ} \mathrm{X}$ peaks can only be observed in highly crystalline homoepitaxial GaN samples; thus, the electrochemical etching process used to form NP GaN does not degrade the crystal quality of GaN. ${ }^{53,54}$ It should also be noted that the PL spectrum of the planar GaN layer sample and that of the NP GaN sample without 
$\mathrm{Ga}_{2} \mathrm{O}_{3}$ displayed several interesting features. For instance, a secondary peak around the dominant peak ( $\mathrm{D}^{\circ} \mathrm{X} \sim 3.4 \mathrm{eV}$ ), attributed to a basal-plane stacking fault (bSF), was observed in the two samples. ${ }^{55}$ The bSF emission peak is a well-known intrinsic defect peak and is commonly observed in epitaxially grown GaN. Other defect-related transition lines of ultraviolet luminescence (UVL) near $3.3 \mathrm{eV}$ and blue luminescence (BL) around 2.9-3.0 eV were also observed. ${ }^{56-58}$ However, these defect-related bSF, UVL, and BL peak were only observed in the planar GaN layer sample and NP GaN sample without $\mathrm{Ga}_{2} \mathrm{O}_{3}$. We speculate that defect-related peaks are effectively suppressed by passivation with a thin $\mathrm{Ga}_{2} \mathrm{O}_{3}$ layer surrounding NP GaN.

To clarify the passivation effect of the $\mathrm{Ga}_{2} \mathrm{O}_{3}$ interlayer between the perovskite and NP GaN layers of the heterostructure, we spin-coated $\mathrm{MAPbI}_{3}$ perovskite materials onto two NP GaN samples (with and without $\mathrm{Ga}_{2} \mathrm{O}_{3}$ ), and time-resolved PL (TR-PL) with steady-state PL measurements were carried out on both samples at room temperature (Figure 3e). The TR-PL spectrum of $\mathrm{MAPbI}_{3}$ on NP GaN with $\mathrm{Ga}_{2} \mathrm{O}_{3}$ showed a relatively short PL average lifetime $(t=$ $10.07 \mathrm{~ns})$, while $\mathrm{MAPbI}_{3}$ on $\mathrm{NP} \mathrm{GaN}$ without $\mathrm{Ga}_{2} \mathrm{O}_{3}$ exhibited a longer PL lifetime $(t=18.20 \mathrm{~ns}$, see Table S1). We attribute such short lifetime to the presence of $\mathrm{Ga}_{2} \mathrm{O}_{3}$ between the $\mathrm{MAPbI}_{3}$ and NP GaN layers, which facilitates the carrier extraction to the $n$-GaN. In the same vein, steady-state PL measurements indicated more pronounced PL quenching of $\mathrm{MAPbI}_{3}$ on NP GaN with $\mathrm{Ga}_{2} \mathrm{O}_{3}$ than on that without $\mathrm{Ga}_{2} \mathrm{O}_{3}$. Thus, introducing the $\mathrm{Ga}_{2} \mathrm{O}_{3}$ interlayer is a useful strategy to suppress non-radiative recombination that occurs as a consequence of defect-related deep levels and energyband mismatching, as shown in Figure 3f.

To further evaluate the effects of different porosities on NP GaN/MAPbI 3 PSCs, we compared the corresponding current-voltage characteristics of the samples using two solar cell characterization methods: $J-V$ and EQE measurements. Figure 3g shows the current-voltage 
characteristics of our best-performing $\mathrm{MAPbI}_{3}$ PSCs with planar $n$-GaN layer and NP GaN structures. The reverse-scan short-circuit current density $\left(J_{\mathrm{SC}}\right)$, open-circuit voltage $\left(V_{\mathrm{OC}}\right)$, and fill factor $(F F)$ of the solar cell containing planar $n-\mathrm{GaN}$ were $11.16 \mathrm{~mA} / \mathrm{cm}^{2}, 0.99 \mathrm{~V}$, and $46.1 \%$, respectively. The rigid PSCs containing a planar $n$-GaN layer exhibited a best PCE of 5.09\%. In contrast, the $\mathrm{MAPbI}_{3}$ PSCs containing NP GaN showed a considerable enhancement in $J_{\mathrm{SC}}, V_{\mathrm{OC}}$, and $F F$ (see Table 1). The PCE of the solar cells increased continuously as $\rho_{\mathrm{s}}$ was increased from $7.2 \%$ to $47.5 \%$ and to $71.7 \%$. When the $\rho_{\mathrm{s}}$ was increased to $71.7 \%$, the rigid PSCs exhibited the best PCE of $18.79 \%$. The corresponding external quantum efficiency (EQE) and integrated $J_{\mathrm{SC}}$ of a representative solar cell containing NP GaN $\left(\rho_{\mathrm{s}}=71.7 \%\right)$ is shown in Figure 3h. Figure S4 shows the detailed performance characteristics of our PSCs with NP GaN $\left(\rho_{\mathrm{s}}=71.7 \%\right)$. To further understand the influence of the $\mathrm{Ga}_{2} \mathrm{O}_{3}$ interlayer, we also fabricated solar cells from which the $\mathrm{Ga}_{2} \mathrm{O}_{3}$ interlayer of the NP GaN structure was removed by chemical etching with $\mathrm{BOE}$ at room temperature for 5 min (Figure 3i). We observed that the presence of the $\mathrm{Ga}_{2} \mathrm{O}_{3}$ interlayer between the NP GaN and perovskite layers, which formed spontaneously at the NP GaN surface during the electrochemical etching process, contributes to the increased solar cell efficiency. Our PSC with NP GaN/n-GaN exhibits the highest efficiency among published ITO-free PSCs with alternative TCLs (see Table S3).

To investigate the light absorption efficiency of the NP GaN/MAPbI 3 , we performed FDTD simulations. Figure 4a illustrates the simulated structure of the PSC with $\mathrm{NP} \mathrm{GaN} / \mathrm{MAPbI}_{3}$ under UV and visible light illumination $(\lambda=350 \mathrm{~nm}-800 \mathrm{~nm})$. The dispersion and extinction coefficients of each material $\left(\mathrm{GaN}\right.$ and $\left.\mathrm{MAPbI}_{3}\right)$ were taken from the reported values in the literature. ${ }^{59,60}$ To model the effects of the nanopore distribution in the NP GaN, we designed the NP GaN in the FDTD simulations as repeating planes of porosity, $\rho_{s}$, such that the porosity per 
unit volume, $\rho_{\mathrm{v}}$ is equivalent to its experimentally estimated value. Figure $\mathbf{4 b}$ shows the contrast difference in effective refractive index $\left(n_{\text {eff }}\right)$ of NP GaN/MAPbI $3\left(\rho_{\mathrm{s}}=71.7 \%\right)$. The simulated $n_{\text {eff }}$ of $\mathrm{NP} \mathrm{GaN} / \mathrm{MAPbI}_{3}$ shows an intermediate value between $\mathrm{GaN}$ 's and $\mathrm{MAPbI}_{3}$ 's intrinsic refractive indices at the all incident lights $(\lambda=350 \mathrm{~nm}-800 \mathrm{~nm})$, because the two homogeneously mixed materials follow the $n_{\text {eff }}$ based on the 2D Maxwell-Garnett theory ${ }^{61,62}$ (see the Supporting Information for details). According to the FDTD simulations, Fresnel reflections are significantly suppressed in a PSC structure sequentially consisting of $2.0 \mu \mathrm{m}$-thick $n$-GaN, NP GaN/MAPbI ${ }_{3}$, and $\mathrm{MAPbI}_{3}$ with a gradual refractive index change, compared to those of a PSC structure having an abrupt interface; and, thus, decreasing photon losses.

Since UV light photocatalytically degrades the $\mathrm{MAPbI}_{3}{ }^{63,64}$ - and blocking it increases the lifespan of PSCs - we investigated the propagation UV light $(\lambda=350 \mathrm{~nm})$ in the simulated PSC structure. Figure 4c shows that a $2.0 \mu \mathrm{m}$-thick $n$-GaN layer blocks incident UV light entirely from reaching the NP GaN/MAPbI 3 or $\mathrm{MAPbI}_{3}$ perovskite light absorber layers.

In contrast, light within the visible spectrum $(\lambda=400,530$, and $660 \mathrm{~nm})$ penetrated the PSC structures, and was only absorbed by the perovskite within the $\mathrm{MAPbI}_{3}$ and $\mathrm{NP} \mathrm{GaN} / \mathrm{MAPbI}_{3}$ layers (see Figure 4d for the simulated absorption profiles). Interestingly, the PSC with NP $\mathrm{GaN} / \mathrm{MAPbI}_{3}$ shows an extended absorption distribution along the entire NP GaN/MAPbI${ }_{3}$, implying that the hierarchical configuration of $\mathrm{NP} \mathrm{GaN} / \mathrm{MAPbI} \mathrm{I}_{3}$ further increases the probability of light absorption.

To analyze the porosity- and thickness-dependent light absorption efficiencies, we performed RCWA simulations. Figure 4e shows the contour maps of calculated light absorptance $(\lambda=400-$ $800 \mathrm{~nm}$ ) by increasing the thickness of NP GaN//MAPbI 3 and the porosity of NP GaN. The two 
dashed white lines demarcate the region of the graph for $80 \%$ and $90 \%$ of the incident light absorptance according to the porosity of NP GaN. This result reveals that when the porosity of NP GaN is between $10 \%$ to $80 \%$, the structure shows high absorption for visible light. Moreover, when the $\mathrm{NP} \mathrm{GaN} / / \mathrm{MAPbI}_{3}$ light absorber has a thickness of more than $2.0 \mu \mathrm{m}$, perfect absorption occurs throughout the visible light regions. Figure $4 \mathbf{f}$ shows the average light absorptance of 0.1 and 2.0 $\mu$ m-thick NP GaN/MAPbI ${ }_{3}$ across the entire visible wavelengths. The simulation results suggest that the optimal porosity of NP GaN and the optimal thickness of the NP GaN/MAPbI 3 should be between $10 \%$ to $80 \%$ and $\geq 2.0 \mu \mathrm{m}$, respectively.

In conclusion, the cathode structure in a PSC plays a key role in the extraction of photogenerated electrons. Therefore, one of the most critical aspects of PSC fabrication is finding a suitable combination of a TCL and an ETL, which are typically comprised of differing materials, to form the cathode structure. In this respect, we proposed a novel cathode structure for PSCs, a NP GaN $/ n$-GaN that combines the function of both the TCL and ETL. The NP GaN/n-GaN structure affords enhanced light harvesting (as a result of the structure's gradual refractive index change) and efficient carrier extraction due to the enlarged perovskite interface with the cathode. A $\mathrm{Ga}_{2} \mathrm{O}_{3}$ interlayer (formed spontaneously at the surface of the NP GaN layer during the electrochemical etching process) between $\mathrm{NP}$ GaN and perovskite contributes to interface passivation. These advantageous properties enabled the fabrication of $\mathrm{MAPbI}_{3} \mathrm{PSCs}$ based on NP $\mathrm{GaN} / n$-GaN structures that show promising PCEs of up to $18.79 \%$. Our work opens an avenue for PSCs to benefit from the versatile heterostructures afforded by the mature III-nitride-based technologies. 
a
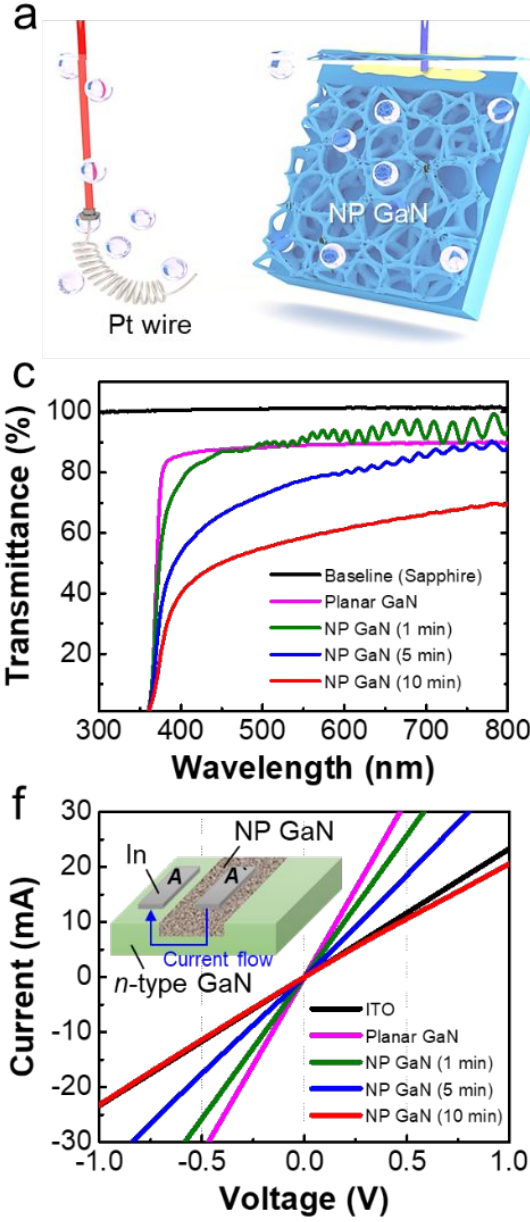

b

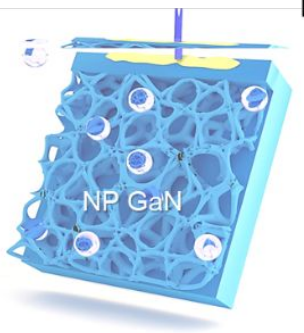

d
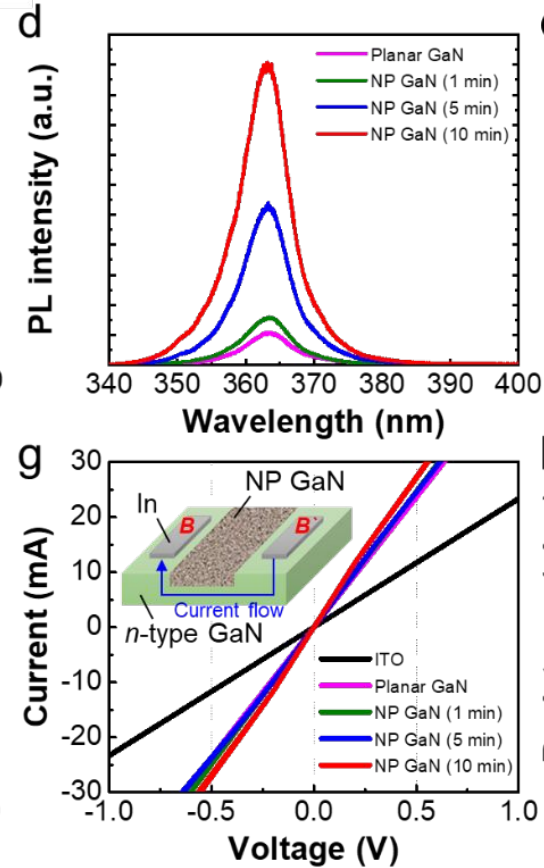

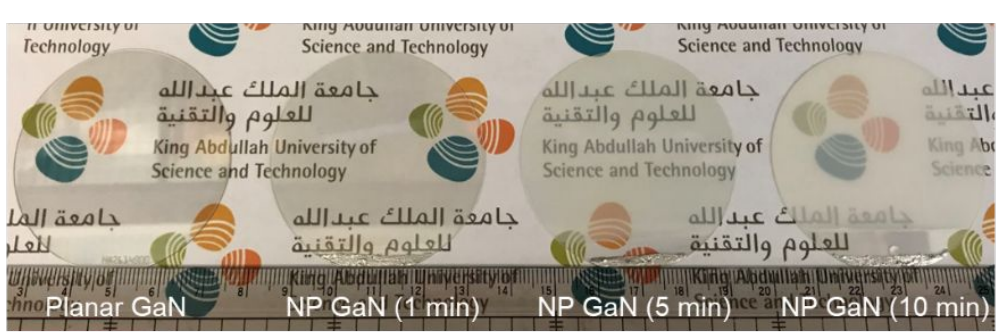

e
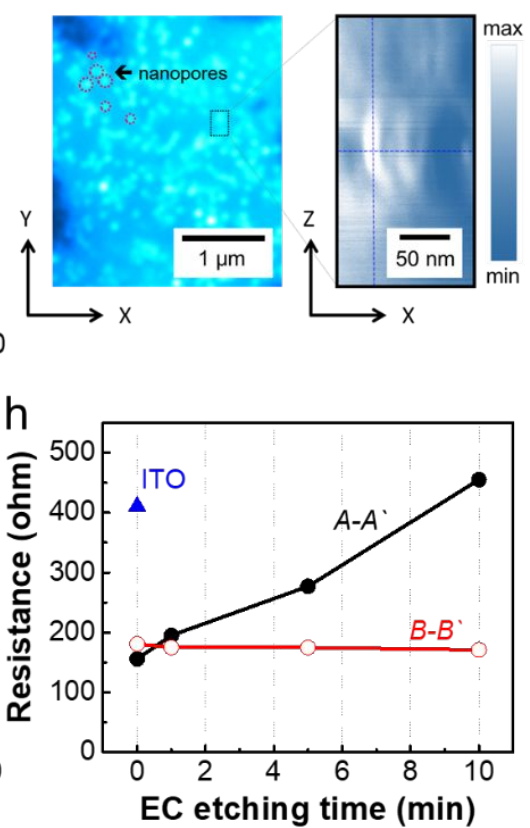

Figure 1. (a) Schematic diagram of electrochemical etching process for NP GaN. (b) Photographs of NP GaN obtained at various electrochemical etching times. (c) Transmittance of NP GaN as a function of electrochemical etching time. (d) PL spectra of NP GaN as a function of electrochemical etching time. (e) Confocal PL images of photons generated by nanopore-induced Rayleigh scattering. Current-voltage curve (f) through the NP GaN region; here the current flow through NP GaN is denoted as A-A', (g) lateral electrical transport under the NP GaN layer (denoted as B-B'), and (h) resistances of NP GaN as a function of electrochemical etching time. 


\section{a \\ b}
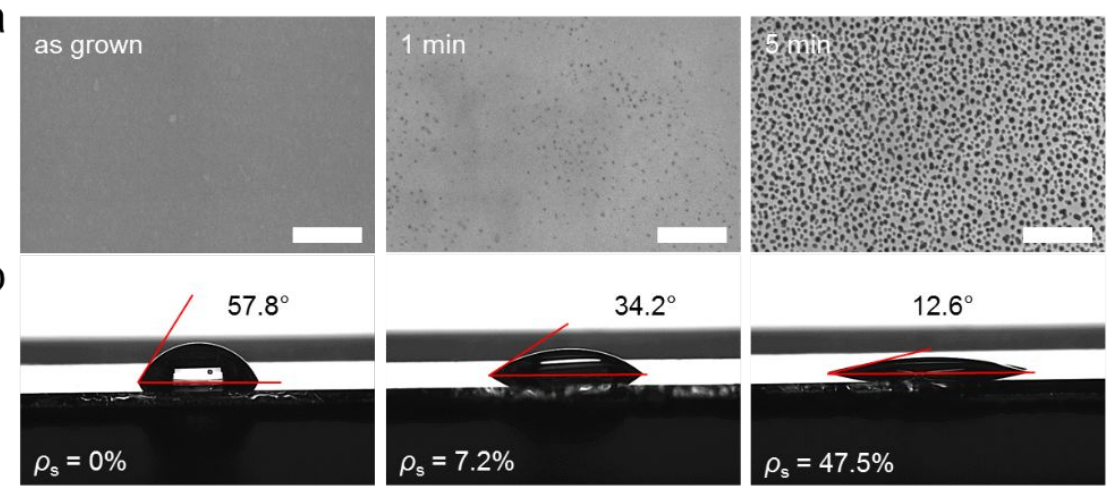

C
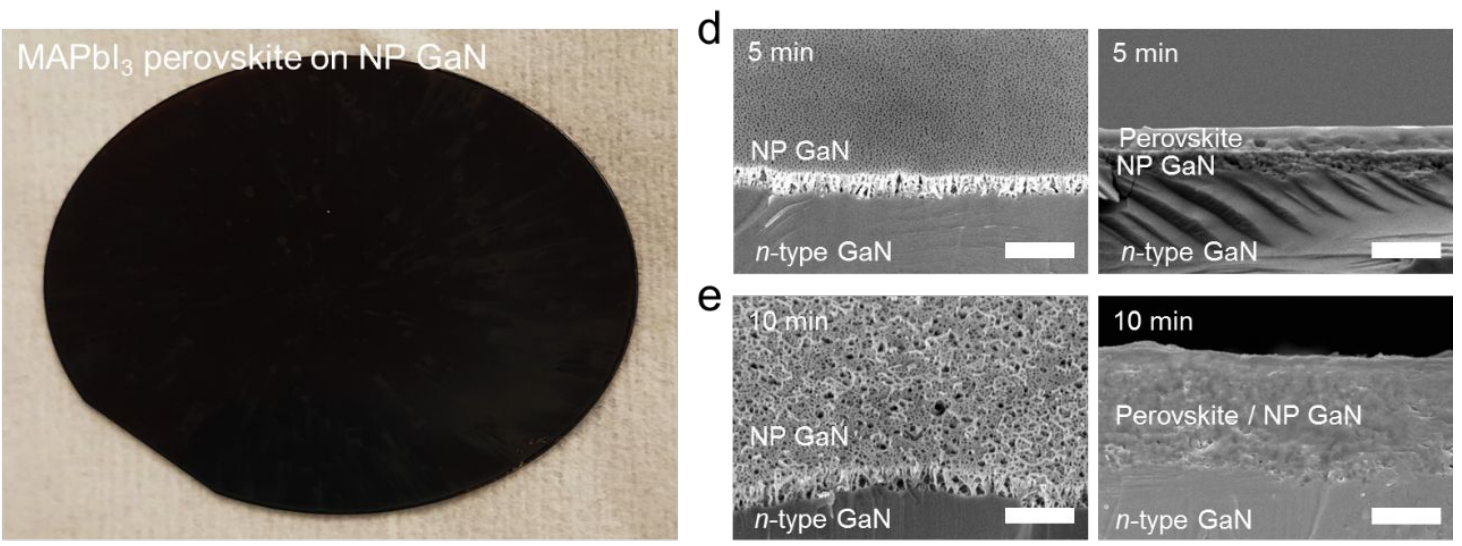

Figure 2. Plan-view scanning electron microscopy (SEM) images of (a) $n$-GaN layer template and $\mathrm{NP} \mathrm{GaN}$ formed after $1 \mathrm{~min}, 5 \mathrm{~min}$, and 10 min of electrochemical etching. Scale bar is $500 \mathrm{~nm}$. (b) Water contact angle measurement for $n$-GaN template with NP GaN formed after $1 \mathrm{~min}, 5 \mathrm{~min}$, and $10 \mathrm{~min}$ of electrochemical etching. (c) Photograph of perovskite $\mathrm{MAPbI}_{3}$ spin-coated thin film on a 2-inch NP GaN wafer. Bird's-eye and cross-sectional SEM images of (d) NP GaN formed after $5 \mathrm{~min}$ and (e) $10 \mathrm{~min}$ of electrochemical etching. Scale bar is $1.0 \mu \mathrm{m}$. 
a

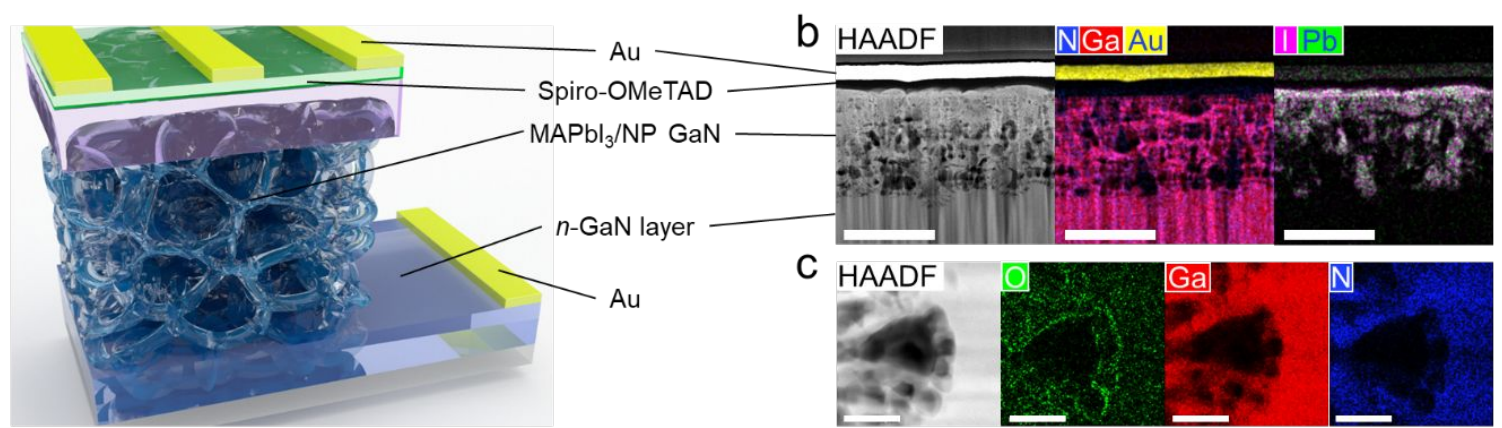

Figure 3. (a) A schematic illustration of the PSC structure including NP GaN/MAPbI 3 . (b) Crosssectional high-angle annular dark-field scanning transmission electron microscopy (HAADFSTEM) image of PSC; electron energy loss spectroscopy (EELS) elemental mapping of N, Ga, and $\mathrm{Au}$ for the structural overview and of $\mathrm{Pb}$ and I confirm that those materials are well incorporated into NP GaN. Scale bar is $1 \mu \mathrm{m}$. (c) Cross-sectional HAADF-STEM image of the NP $\mathrm{GaN} /$ planar GaN interface and EELS elemental mapping of $\mathrm{O}, \mathrm{Ga}$, and N, respectively. Scale bar is $100 \mathrm{~nm}$. (d) Low-temperature $(10 \mathrm{~K}) \mathrm{PL}$ measurements of planar GaN template and NP GaN (with and without $\mathrm{Ga}_{2} \mathrm{O}_{3}$ ) samples for defect study. Comparison of NP GaN with and without $\mathrm{Ga}_{2} \mathrm{O}_{3}$ by (e) normalized transient PL decay profile (left) and steady-state PL (right). (f) Comparison of energy band diagrams for devices with and without $\mathrm{Ga}_{2} \mathrm{O}_{3}$. (g) Current-voltage characteristics of $\mathrm{MAPbI}_{3}$ PSCs with planar GaN layer and NP GaN structures with porosities of $\rho_{\mathrm{s}}=0,7.2,47.5$, and 71.7\%. (h) Incident photon-to-current efficiency of solar cell using NP GaN $\left(\rho_{\mathrm{s}}=71.7 \%\right.$ ) and (i) comparison of current-voltage characteristics for $\mathrm{NP} \mathrm{GaN} / \mathrm{MAPbI}_{3}$ solar cells with and without $\mathrm{Ga}_{2} \mathrm{O}_{3}$ interlayer. 

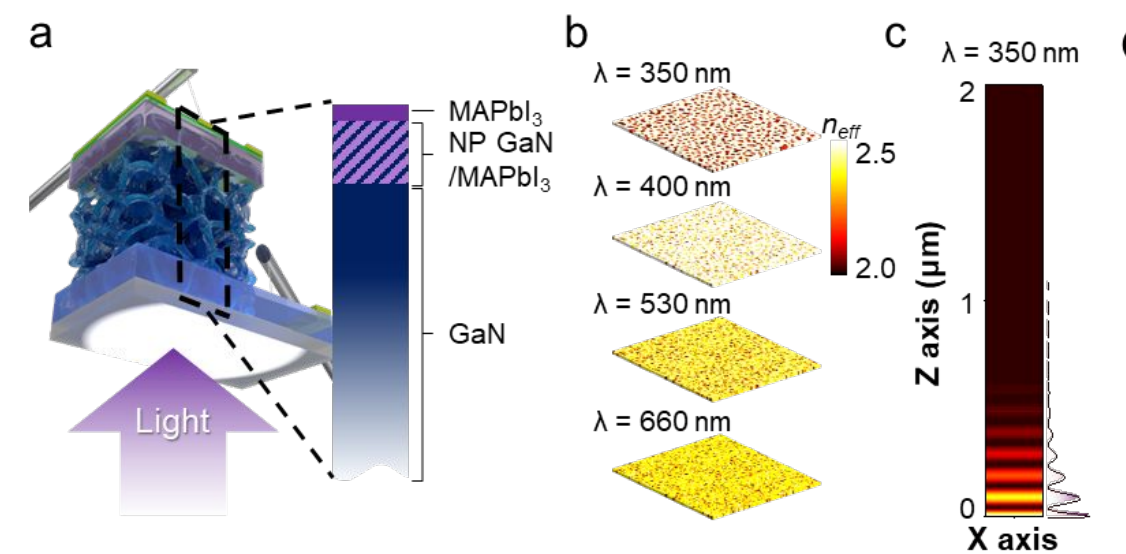

d $\begin{array}{r}\lambda \text { of incident light } \\ 400 \mathrm{~nm} 530 \mathrm{~nm} 660 \mathrm{~nm}\end{array}$

$\rho_{v}=0 \%$
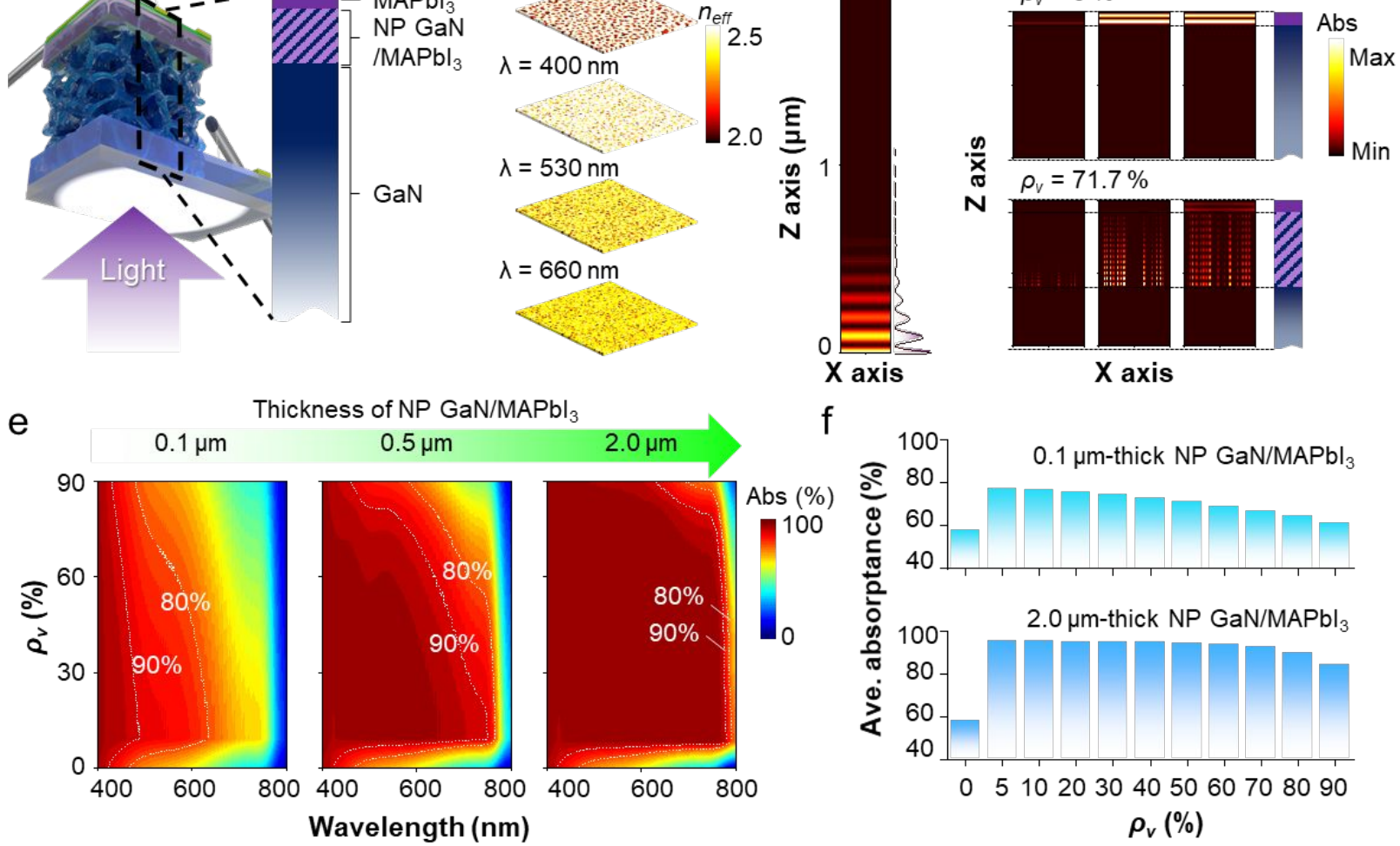

Figure 4. (a) A schematic diagram of the PSC modelled for the FDTD simulation. (b) Contrast difference in effective refractive index $\left(n_{\text {eff }}\right)$ of NP GaN/MAPbI $3\left(\rho_{\mathrm{s}}=71.7 \%\right)$ according to the wavelength of incident light $(\lambda=350,400,530$, and $660 \mathrm{~nm})$. Simulated contour maps of the light absorption for (c) planar $n$-GaN layer with UV incident light $(\lambda=350 \mathrm{~nm})$ and (d) NP $\mathrm{GaN} / \mathrm{MAPbI}_{3}$ on $n$-GaN with white light $\left(\lambda=400,530\right.$, and $660 \mathrm{~nm}$ ). $\rho_{\mathrm{s}}=0 \%$ (top) consists of $\mathrm{MAPbI}_{3}$ on $n-\mathrm{GaN}$ without NP GaN and $\rho_{\mathrm{s}}=71.7 \%$ (bottom) consists of NP GaN/MAPbI 3 on $n-$ $\mathrm{GaN}$ with a volume ratio of NP GaN (28.3\%) and $\mathrm{MAPbI}_{3}$ (71.7\%) filling nanopores of the NP GaN. (e) Simulated contour maps for the absorption spectra of NP GaN/MAPbI ${ }_{3}$ in the visible light region as functions of the porosity and thickness of $\mathrm{NP} \mathrm{GaN} / \mathrm{MAPbI}_{3}$ light absorber. Two dashed lines in contour maps indicate the criteria for $80 \%$ and $90 \%$ of the incident light absorptance. (f) The averaged light absorptance of 0.1 (top) and $2.0 \mu \mathrm{m}$-thick (bottom) NP GaN/MAPbI ${ }_{3}$ with varying $\rho_{\mathrm{v}}$, respectively. 
Table 1. Summary of the $J_{\mathrm{sc}}, V_{\mathrm{oc}}, F F$, and PCE parameters for PSCs with planar GaN and NP GaN.

\begin{tabular}{ccccc}
\hline & $\boldsymbol{J}_{\mathrm{sc}}$ & $\boldsymbol{V}_{\text {oc }}$ & $\boldsymbol{F F}$ & PCE \\
\hline Planar GaN $\left(\rho_{\mathrm{s}}=0 \%\right)$ & $11.16 \mathrm{~mA} / \mathrm{cm}^{2}$ & $0.99 \mathrm{~V}$ & $46.1 \%$ & $5.09 \%$ \\
NP GaN $\left(\rho_{\mathrm{s}}=7.2 \%\right)$ & $19.17 \mathrm{~mA} / \mathrm{cm}^{2}$ & $0.99 \mathrm{~V}$ & $67.2 \%$ & $12.75 \%$ \\
$\mathrm{NPGaN}\left(\rho_{\mathrm{s}}=47.5 \%\right)$ & $21.08 \mathrm{~mA} / \mathrm{cm}^{2}$ & $1.05 \mathrm{~V}$ & $72.8 \%$ & $16.12 \%$ \\
$\mathrm{NP} \mathrm{GaN}\left(\rho_{\mathrm{s}}=71.7 \%\right)$ & $22.87 \mathrm{~mA} / \mathrm{cm}^{2}$ & $1.07 \mathrm{~V}$ & $76.8 \%$ & $18.79 \%$ \\
\hline
\end{tabular}




\section{ASSOCIATED CONTENT}

Supporting Information. Experimental details of $n$-GaN growth, electrochemical etching process, TEM, device fabrication, current-voltage characteristics, EQE measurement, FDTD simulation, PL, and TRPL.

\section{AUTHOR INFORMATION}

\section{Notes}

The authors declare no competing financial interest.

\section{ACKNOWLEDGMENT}

The authors gratefully acknowledge the financial support provided by King Abdullah University of Science and Technology (KAUST). 


\section{References}

(1) Zheng, X.; Hou, Y.; Bao, C.; Yin, J.; Yuan, F.; Huang, Z.; Song, K.; Liu, J.; Troughton, J.; Gasparini, N. Managing Grains and Interfaces via Ligand Anchoring Enables 22.3\%Efficiency Inverted Perovskite Solar Cells. Nat. Energy 2020, 5 (2), 131-140.

(2) Zheng, X.; Troughton, J.; Gasparini, N.; Lin, Y.; Wei, M.; Hou, Y.; Liu, J.; Song, K.; Chen, Z.; Yang, C. Quantum Dots Supply Bulk-and Surface-Passivation Agents for Efficient and Stable Perovskite Solar Cells. Joule 2019, 3 (8), 1963-1976.

(3) Green, M. A.; Ho-Baillie, A.; Snaith, H. J. The Emergence of Perovskite Solar Cells. Nat. Photonics 2014, 8 (7), 506.

(4) Arora, N.; Dar, M. I.; Hinderhofer, A.; Pellet, N.; Schreiber, F.; Zakeeruddin, S. M.; Grätzel, M. Perovskite Solar Cells with CuSCN Hole Extraction Layers Yield Stabilized Efficiencies Greater than 20\%. Science (80-. ). 2017, 358 (6364), 768-771.

(5) Saliba, M.; Matsui, T.; Seo, J.-Y.; Domanski, K.; Correa-Baena, J.-P.; Nazeeruddin, M. K.; Zakeeruddin, S. M.; Tress, W.; Abate, A.; Hagfeldt, A. Cesium-Containing Triple Cation Perovskite Solar Cells: Improved Stability, Reproducibility and High Efficiency. Energy Environ. Sci. 2016, 9 (6), 1989-1997.

(6) Wang, Y.; Dar, M. I.; Ono, L. K.; Zhang, T.; Kan, M.; Li, Y.; Zhang, L.; Wang, X.; Yang, Y.; Gao, X. Thermodynamically Stabilized $\beta$-CsPbI3-Based Perovskite Solar Cells with Efficiencies $>18 \%$. Science (80-. ). 2019, 365 (6453), 591-595.

(7) Chen, R.; Cao, J.; Duan, Y.; Hui, Y.; Chuong, T. T.; Ou, D.; Han, F.; Cheng, F.; Huang, X.; Wu, B. High-Efficiency, Hysteresis-Less, UV-Stable Perovskite Solar Cells with Cascade ZnO-ZnS Electron Transport Layer. J. Am. Chem. Soc. 2018, 141 (1), 541-547.

(8) Nie, W.; Tsai, H.; Asadpour, R.; Blancon, J.-C.; Neukirch, A. J.; Gupta, G.; Crochet, J. J.; Chhowalla, M.; Tretiak, S.; Alam, M. A. High-Efficiency Solution-Processed Perovskite Solar Cells with Millimeter-Scale Grains. Science (80-. ). 2015, 347 (6221), 522-525.

(9) Chen, Z.; Turedi, B.; Alsalloum, A. Y.; Yang, C.; Zheng, X.; Gereige, I.; AlSaggaf, A.; Mohammed, O. F.; Bakr, O. M. Single-Crystal MAPbI3 Perovskite Solar Cells Exceeding 21\% Power Conversion Efficiency. ACS Energy Lett. 2019, 4 (6), 1258-1259.

(10) Alsalloum, A. Y.; Turedi, B.; Zheng, X.; Mitra, S.; Zhumekenov, A. A.; Lee, K. J.; Maity, P.; Gereige, I.; AlSaggaf, A.; Roqan, I. S. Low-Temperature Crystallization Enables 21.9\% Efficient Single-Crystal MAPbI3 Inverted Perovskite Solar Cells. ACS Energy Lett. 2020, $5(2), 657-662$.

(11) Liu, M.; Johnston, M. B.; Snaith, H. J. Efficient Planar Heterojunction Perovskite Solar Cells by Vapour Deposition. Nature 2013, 501 (7467), 395-398.

(12) Lee, K. J.; Turedi, B.; Sinatra, L.; Zhumekenov, A. A.; Maity, P.; Dursun, I.; Naphade, R.; Merdad, N.; Alsalloum, A.; Oh, S. Perovskite-Based Artificial Multiple Quantum Wells. Nano Lett. 2019, 19 (6), 3535-3542. 
(13) Turren-Cruz, S.-H.; Hagfeldt, A.; Saliba, M. Methylammonium-Free, High-Performance, and Stable Perovskite Solar Cells on a Planar Architecture. Science (80-. ). 2018, 362 (6413), 449-453.

(14) Qiu, L.; He, S.; Ono, L. K.; Liu, S.; Qi, Y. Scalable Fabrication of Metal Halide Perovskite Solar Cells and Modules. ACS Energy Lett. 2019, 4 (9), 2147-2167.

(15) Carlson, D. E.; Wronski, C. R. Amorphous Silicon Solar Cell. Appl. Phys. Lett. 1976, 28 (11), 671-673.

(16) Hamaker, H. C.; Ford, C. W.; Werthen, J. G.; Virshup, G. F.; Kaminar, N. R.; King, D. L.; Gee, J. M. 26\% Efficient Magnesium-doped AlGaAs/GaAs Solar Concentrator Cells. Appl. Phys. Lett. 1985, 47 (7), 762-764.

(17) Shin, I.-S.; Kim, J.; Lee, D.; Kim, D.; Park, Y.; Yoon, E. Epitaxial Growth of SingleCrystalline AlN Layer on Si (111) by DC Magnetron Sputtering at Room Temperature. Jpn. J. Appl. Phys. 2018, 57 (6), 60306.

(18) Singh, R.; Giri, A.; Pal, M.; Thiyagarajan, K.; Kwak, J.; Lee, J.-J.; Jeong, U.; Cho, K. Perovskite Solar Cells with an MoS 2 Electron Transport Layer. J. Mater. Chem. A 2019, 7 (12), 7151-7158.

(19) Wang, Y.; Wan, J.; Ding, J.; Hu, J.; Wang, D. A Rutile TiO2 Electron Transport Layer for the Enhancement of Charge Collection for Efficient Perovskite Solar Cells. Angew. Chemie Int. Ed. 2019, 58 (28), 9414-9418.

(20) Son, D.-Y.; Im, J.-H.; Kim, H.-S.; Park, N.-G. 11\% Efficient Perovskite Solar Cell Based on ZnO Nanorods: An Effective Charge Collection System. J. Phys. Chem. C 2014, 118 (30), 16567-16573.

(21) Chen, Y.; Meng, Q.; Zhang, L.; Han, C.; Gao, H.; Zhang, Y.; Yan, H. SnO2-Based Electron Transporting Layer Materials for Perovskite Solar Cells: A Review of Recent Progress. J. Energy Chem. 2019, 35, 144-167.

(22) Guo, H.; Chen, H.; Zhang, H.; Huang, X.; Yang, J.; Wang, B.; Li, Y.; Wang, L.; Niu, X.; Wang, Z. Low-Temperature Processed Yttrium-Doped SrSnO3 Perovskite Electron Transport Layer for Planar Heterojunction Perovskite Solar Cells with High Efficiency. Nano Energy 2019, 59, 1-9.

(23) Zhu, Z.; Zhao, D.; Chueh, C.-C.; Shi, X.; Li, Z.; Jen, A. K.-Y. Highly Efficient and Stable Perovskite Solar Cells Enabled by All-Crosslinked Charge-Transporting Layers. Joule 2018, 2 (1), 168-183.

(24) Mahmood, K.; Sarwar, S.; Mehran, M. T. Current Status of Electron Transport Layers in Perovskite Solar Cells: Materials and Properties. RSC Adv. 2017, 7 (28), 17044-17062.

(25) Tavakoli, M. M.; Tavakoli, R.; Yadav, P.; Kong, J. A Graphene/ZnO Electron Transfer Layer Together with Perovskite Passivation Enables Highly Efficient and Stable Perovskite Solar Cells. J. Mater. Chem. A 2019, 7 (2), 679-686. 
(26) Xiong, L.; Guo, Y.; Wen, J.; Liu, H.; Yang, G.; Qin, P.; Fang, G. Review on the Application of SnO2 in Perovskite Solar Cells. Adv. Funct. Mater. 2018, 28 (35), 1802757.

(27) Docampo, P.; Ball, J. M.; Darwich, M.; Eperon, G. E.; Snaith, H. J. Efficient Organometal Trihalide Perovskite Planar-Heterojunction Solar Cells on Flexible Polymer Substrates. Nat. Commun. 2013, 4 (1), 1-6.

(28) Yu, H.; Lee, J. W.; Yun, J.; Lee, K.; Ryu, J.; Lee, J.; Hwang, D.; Kim, S. K.; Jang, J. Outstanding Performance of Hole-Blocking Layer-Free Perovskite Solar Cell Using Hierarchically Porous Fluorine-Doped Tin Oxide Substrate. Adv. Energy Mater. 2017, 7 (22), 1700749.

(29) Ye, S.; Rathmell, A. R.; Chen, Z.; Stewart, I. E.; Wiley, B. J. Metal Nanowire Networks: The next Generation of Transparent Conductors. Adv. Mater. 2014, 26 (39), 6670-6687.

(30) Service, R. F. Nations Move to Head off Shortages of Rare Earths. American Association for the Advancement of Science 2010.

(31) Nakamura, E.; Sato, K. Managing the Scarcity of Chemical Elements. Nat. Mater. 2011, 10 (3), 158-161.

(32) Poncé, S.; Jena, D.; Giustino, F. Route to High Hole Mobility in GaN via Reversal of Crystal-Field Splitting. Phys. Rev. Lett. 2019, 123 (9), 96602.

(33) Levinshtein, M. E.; Rumyantsev, S. L.; Shur, M. S. Properties of Advanced Semiconductor Materials: GaN, AIN, InN, BN, SiC, SiGe; John Wiley \& Sons, 2001.

(34) Lee, K. J.; Kim, S.-J.; Kim, J.-J.; Hwang, K.; Kim, S.-T.; Park, S.-J. Enhanced Performance of InGaN/GaN Multiple-Quantum-Well Light-Emitting Diodes Grown on Nanoporous GaN Layers. Opt. Express 2014, 22 (104), A1164-A1173.

(35) Prabaswara, A.; Min, J.-W.; Zhao, C.; Janjua, B.; Zhang, D.; Albadri, A. M.; Alyamani, A. Y.; Ng, T. K.; Ooi, B. S. Direct Growth of III-Nitride Nanowire-Based Yellow LightEmitting Diode on Amorphous Quartz Using Thin Ti Interlayer. Nanoscale Res. Lett. 2018, $13(1), 1-9$.

(36) Schmidt, M. C.; Kim, K.-C.; Farrell, R. M.; Feezell, D. F.; Cohen, D. A.; Saito, M.; Fujito, K.; Speck, J. S.; DenBaars, S. P.; Nakamura, S. Demonstration of Nonpolar M-Plane InGaN/GaN Laser Diodes. Jpn. J. Appl. Phys. 2007, 46 (3L), L190.

(37) Binet, F.; Duboz, J. Y.; Rosencher, E.; Scholz, F.; Härle, V. Mechanisms of Recombination in GaN Photodetectors. Appl. Phys. Lett. 1996, 69 (9), 1202-1204.

(38) Lim, K. T. P.; Deakin, C.; Ding, B.; Bai, X.; Griffin, P.; Zhu, T.; Oliver, R. A.; Credgington, D. Encapsulation of Methylammonium Lead Bromide Perovskite in Nanoporous GaN. APL Mater. 2019, 7 (2), 21107.

(39) Zhou, H.; Mei, J.; Xue, M.; Song, Z.; Wang, H. High-Stability, Self-Powered Perovskite Photodetector Based on a $\mathrm{CH} 3 \mathrm{NH} 3 \mathrm{PbI} / \mathrm{GaN}$ Heterojunction with $\mathrm{C} 60$ as an Electron Transport Layer. J. Phys. Chem. C 2017, 121 (39), 21541-21545. 
(40) Gong, J.; Liu, S.; He, Y.; Feng, X.; Xia, X.; Quan, Z.; Wang, L. Study on the Band Alignment of GaN/CH3NH3PbBr3 Heterojunction by x-Ray Photoelectron Spectroscopy. Appl. Phys. Lett. 2017, 111 (12), 122103.

(41) Wang, Y.; Zheng, D.; Li, L.; Zhang, Y. Enhanced Efficiency of Flexible GaN/Perovskite Solar Cells Based on the Piezo-Phototronic Effect. ACS Appl. Energy Mater. 2018, 1 (7), 3063-3069.

(42) Chen, S.; Zhang, C.; Lee, J.; Han, J.; Nurmikko, A. High-Q, Low-Threshold Monolithic Perovskite Thin-Film Vertical-Cavity Lasers. Adv. Mater. 2017, 29 (16), 1604781.

(43) Nakamura, S.; Harada, Y.; Seno, M. Novel Metalorganic Chemical Vapor Deposition System for GaN Growth. Appl. Phys. Lett. 1991, 58 (18), 2021-2023.

(44) Fujito, K.; Kubo, S.; Nagaoka, H.; Mochizuki, T.; Namita, H.; Nagao, S. Bulk GaN Crystals Grown by HVPE. J. Cryst. Growth 2009, 311 (10), 3011-3014.

(45) Kočan, M.; Rizzi, A.; Lüth, H.; Keller, S.; Mishra, U. K. Surface Potential at As-Grown GaN (0001) MBE Layers. Phys. status solidi 2002, 234 (3), 773-777.

(46) Kim, D. H.; Kumar, V.; Chen, G.; Dabiran, A. M.; Wowchak, A. M.; Osinsky, A.; Adesida, I. ALD A12O3 Passivated MBE-Grown AlGaN/GaN HEMTs on 6H-SiC. Electron. Lett. 2007, 43 (2), 127-128.

(47) Nonomura, S.; Kobayashi, S.; Gotoh, T.; Hirata, S.; Ohmori, T.; Itoh, T.; Nitta, S.; Morigaki, K. Photoconductive A-GaN Prepared by Reactive Sputtering. J. Non. Cryst. Solids 1996, 198, 174-177.

(48) Choi, J. H.; Zoulkarneev, A.; Kim, S. Il; Baik, C. W.; Yang, M. H.; Park, S. S.; Suh, H.; Kim, U. J.; Son, H. Bin; Lee, J. S. Nearly Single-Crystalline GaN Light-Emitting Diodes on Amorphous Glass Substrates. Nat. Photonics 2011, 5 (12), 763-769.

(49) Zou, C. W.; Wang, H. J.; Yin, M. L.; Li, M.; Liu, C. S.; Guo, L. P.; Fu, D. J.; Kang, T. W. Preparation of GaN Films on Glass Substrates by Middle Frequency Magnetron Sputtering. J. Cryst. Growth 2009, 311 (2), 223-227.

(50) Lye, K. S.; Kobayashi, A.; Ueno, K.; Ohta, J.; Fujioka, H. InN Thin-Film Transistors Fabricated on Polymer Sheets Using Pulsed Sputtering Deposition at Room Temperature. Appl. Phys. Lett. 2016, 109 (3), 32106.

(51) Cai, J.; Wei, W.; Hu, X.; Wood, D. A. Electrical Conductivity Models in Saturated Porous Media: A Review. Earth-Science Rev. 2017, 171, 419-433.

(52) Lee, K. J.; Chun, J.; Kim, S.-J.; Oh, S.; Ha, C.-S.; Park, J.-W.; Lee, S.-J.; Song, J.-C.; Baek, J. H.; Park, S.-J. Enhanced Optical Output Power of InGaN/GaN Light-Emitting Diodes Grown on a Silicon (111) Substrate with a Nanoporous GaN Layer. Opt. Express 2016, 24 (5), 4391-4398.

(53) Viswanath, A. K.; Lee, J. I.; Yu, S.; Kim, D.; Choi, Y.; Hong, C. Photoluminescence Studies of Excitonic Transitions in GaN Epitaxial Layers. J. Appl. Phys. 1998, 84 (7), 3848-3859. 
(54) Monemar, B. Bound Excitons in GaN. J. Phys. Condens. Matter 2001, 13 (32), 7011.

(55) Forsberg, M.; Serban, A.; Poenaru, I.; Hsiao, C.-L.; Junaid, M.; Birch, J.; Pozina, G. Stacking Fault Related Luminescence in GaN Nanorods. Nanotechnology 2015, 26 (35), 355203.

(56) Reshchikov, M. A. Determination of Acceptor Concentration in GaN from Photoluminescence. Appl. Phys. Lett. 2006, 88 (20), 202104.

(57) Reshchikov, M. A.; Morkoç, H. Luminescence from Defects in GaN. Phys. B Condens. Matter 2006, 376, 428-431.

(58) Reshchikov, M. A.; Morkoç, H. Luminescence Properties of Defects in GaN. J. Appl. Phys. 2005, 97 (6), 5-19.

(59) Kim, Y. J.; Lee, G. J.; Kim, S.; Min, J.-W.; Jeong, S. Y.; Yoo, Y. J.; Lee, S.; Song, Y. M. Efficient Light Absorption by GaN Truncated Nanocones for High Performance Water Splitting Applications. ACS Appl. Mater. Interfaces 2018, 10 (34), 28672-28678.

(60) Leguy, A. M. A.; Azarhoosh, P.; Alonso, M. I.; Campoy-Quiles, M.; Weber, O. J.; Yao, J.; Bryant, D.; Weller, M. T.; Nelson, J.; Walsh, A. Experimental and Theoretical Optical Properties of Methylammonium Lead Halide Perovskites. Nanoscale 2016, 8 (12), 63176327.

(61) Hutchinson, N. J.; Coquil, T.; Richman, E. K.; Tolbert, S. H.; Pilon, L. Reflectance of Surfactant-Templated Mesoporous Silica Thin Films: Simulations versus Experiments. Thin Solid Films 2010, 518 (8), 2134-2140.

(62) Hulkkonen, H. H.; Salminen, T.; Niemi, T. Block Copolymer Patterning for Creating Porous Silicon Thin Films with Tunable Refractive Indices. ACS Appl. Mater. Interfaces 2017, 9 (37), 31260-31265.

(63) Li, Y.; Xu, X.; Wang, C.; Ecker, B.; Yang, J.; Huang, J.; Gao, Y. Light-Induced Degradation of CH3NH3PbI3 Hybrid Perovskite Thin Film. J. Phys. Chem. C 2017, 121 (7), 3904-3910.

(64) Abdelmageed, G.; Jewell, L.; Hellier, K.; Seymour, L.; Luo, B.; Bridges, F.; Zhang, J. Z.; Carter, S. Mechanisms for Light Induced Degradation in MAPbI3 Perovskite Thin Films and Solar Cells. Appl. Phys. Lett. 2016, 109 (23), 233905. 